\title{
Reporting of Negative Randomized Trials in Three Major Medical Journals
}

\author{
Gabriel A. Brooks ${ }^{1}$, Daniel W. Bowles ${ }^{1,2}$, Daniel B. Jamieson ${ }^{1}$, Janice V. Huang ${ }^{1}$, Alexandra Smart ${ }^{1}$, \\ Kathleen A. Heist ${ }^{1}$, Traci E. Yamashita ${ }^{1}$, Allan V. Prochazka, ${ }^{1,2}$, Ravi K. Gopal ${ }^{1,2}$ \\ ${ }^{1}$ Department of Medicine, University of Colorado School of Medicine, Aurora, USA; ${ }^{2}$ Denver VA Medical Center, Eastern Colorado \\ Healthcare System, Denver, USA. \\ Email: Ravi.Gopal@va.gov
}

Received December $13^{\text {th }}, 2012$; revised January $15^{\text {th }}, 2013$; accepted January $22^{\text {nd }}, 2013$

\begin{abstract}
Context: In recent years there has been increasing interest on publication bias and on initiatives to decrease bias, including trial registration. Objective: To test whether there has been an increase in reports of randomized control trials (RCT's) with negative outcomes in major journals and to identify factors associated with these reports. Design: Retrospective review of reports of RCT's published in the Journal of the American Medical Association, The Lancet and the New England Journal of Medicine before (2002-'03, pre-registration era) and after (2007-'08, registration era) the institution of mandatory trial registration. Main Outcome Measure: The primary outcome was the proportion of RCT reports with negative outcomes compared across the two eras. Secondary outcome includes other factors affecting publication. Results: We identified 917 reports of RCT's published in the two study eras. No publications in the pre-registration era reported trial registration compared with $94.4 \%$ in the registration era $(\mathrm{p}<0.001)$. There was a non-significant increase in negative trials from the pre-registration to the registration era $(29.1 \%$ vs. $34.1 \%, p=0.10$, OR 1.26 , $95 \%$ CI $0.96-1.67)$. Study characteristics associated with negative outcomes include trials of drugs (OR 1.62, 95\% CI 1.08 - 2.43), procedures or devices (OR $2.08,95 \%$ CI 1.29 - 3.35), explicit identification of a single primary endpoint (OR 1.70, 95\% CI 1.40 - 2.47), and increasing sample size (OR 3.08, 95\% CI 1.78 - 5.34). Non-inferiority study design was associated with a decreased likelihood of a negative outcome (OR $0.13,95 \%$ CI $0.05-0.31)$. Conclusions: The proportion of published RCT reports with negative outcomes in three major medical journals has not significantly increased since the mandatory clinical trial registration policy. The observed prevalence of negative trials is associated with increases in sample size and greater specificity in trial endpoints.
\end{abstract}

Keywords: Clinical Trial Registration; Publication Bias; Randomized Trials; Clinical Trials; Non-Inferiority Trials

\section{Introduction}

Health care professionals rely on up-to-date, comprehensive, and objective data to make clinical, research and policy decisions. However, a growing body of data suggests that the medical literature used to support these decisions is subject to demonstrable publication bias, with publication of study reports depending on character of the results [1]. Trials with statistically significant outcomes are nearly twice as likely to be published and are published more quickly than so-called "negative trials" $[2,3]$. Publication bias has been particularly evident among studies of investigational drugs [4,5], where proprietary interests may lead drug makers to withhold results of negative trials. These flaws in the medical literature skew systematic interpretation of clinical trial results, misrepresent the experiences of patients who altruistically participate in these trials, and jeopardize the health and safety of future patients.

In response to concerns about lack of transparency in clinical trials, the United States Congress passed the Food and Drug Administration Modernization Act in 1997 calling for the creation of a tool to permit public access to information regarding ongoing clinical trials. The National Institutes of Health (NIH) created the clinicaltrials.gov registry in 2000 in response to this mandate. Initially this registry was voluntary and limited to trials involving medications targeted for "serious or life threatening illnesses". Even with this limited scope there was poor compliance with registration of qualifying studies.

In 2004 the International Committee of Medical Journal Editors (the ICMJE, made up of the editors of eleven leading general medical journals) announced that effective September 2005, registration with clinicaltrials.gov 
or a similar registry would be required as a precondition for publication [6]. Before the introduction of the ICM JE's policy there were fewer than 14,000 trials registered at clinicaltrials.gov; within months of the policy's introduction the number of registered trials increased by $73 \%$. [7]. To date, over 136,000 trials from 182 countries are registered at clinicaltrials.gov [8].

The establishment of clinicaltrials.gov by the NIH and the ICMJE's policy of mandatory clinical trial registration were both intended to increase the transparency of clinical research. Based on anecdotal experience, we hypothesized that the ICMJE's mandatory clinical trial registration policy had resulted in a reduction in publication bias by increasing the proportion of clinical trial reports with negative outcomes published in major medical journals. The primary aim of our study was to test whether there has been an increase in reports of randomized control trials (RCT's) with negative outcomes in major journals and to identify factors associated with these report.

\section{Methods}

\subsection{Study Sample}

Our study sample included all reports of RCT's published in the New England Journal of Medicine, The Lancet and the Journal of the American Medical Association during 2002-'03 (preregistration era) and 2007-'08 (registration era). We selected these journals because they represent the three general medicine journals with the highest impact factors [9]. The two eras were chosen to capture publication patterns before and after the institution of mandatory clinical trial registration policies (initiated in 2005). Two-year intervals were chosen to represent each era based on a pilot sample of 50 articles that suggested an $80 \%$ power to detect a $15 \%$ absolute difference between eras in the proportion of published trials with negative outcomes.

Reports of RCT's were identified using the Cochrane Central Register of Controlled Trials (Cochrane Register), a publicly accessible database maintained by the Cochrane Group. We searched using the full journal name in the source field of the Cochrane Register, limiting searches to the years of interest and excluding entries with "comment" in the publication type field. When searching for entries in the Lancet, we excluded entries with "oncology" or "neurology" in the source field to avoid querying the database for RCT reports published in Lancet Oncology and Lancet Neurology.

After our initial search, we excluded entries in the Cochrane Register that were not reports of RCT's (including duplicate entries, entries for editorials and letters to the editor and entries for trials other than RCT's). We defined an RCT as a prospective study randomizing hu- man subjects into two or more groups for comparison of a defined intervention. We excluded cost-effectiveness trials because they have incremental outcomes that cannot be categorized as positive or negative. We excluded phase I and II trials because these studies frequently do not have efficacy outcomes (phase I studies were initially excluded from the ICMJE's mandatory clinical trial registration policy).

\subsection{Data Abstraction}

For each included entry of an RCT report in the Cochrane Register we abstracted descriptive information and assessed the statistical significance of the primary outcome(s). One reviewer abstracted descriptive information from the structured article abstracts within the Cochrane Register. When abstracts provided insufficient information on endpoints we accessed full reports at the on-line sites of the respective journals. Descriptive information abstracted included year and journal of publication, trial registration number, intervention type (specified nonexclusively as drug, procedure/device, surgery, behaveioral, diagnostic or treatment strategy), sample size, number of study groups, use of placebo control, use of a single primary endpoint and use of a non-inferiority endpoint. We considered trials to be registered if any registration number was reported in the abstract.

Two reviewers assessed each RCT report to categorize the primary outcome(s) as positive or negative with respect to statistical significance of the outcome. We defined a statistically significant (positive) result as having a p-value of less than 0.05 or reaching a pre-specified non-inferiority parameter. Studies having more than one primary outcome or without an explicitly identified primary outcome were categorized as positive if any main outcome reported in the abstract was positive. In cases where there was disagreement between the two reviewers regarding the significance of a study outcome (positive vs. negative), study interpretation was labeled as discordant and a committee of three reviewers reached a consensus determination (GB, AP and RG). This study was deemed exempt from review by the Colorado Multiple Institutional Review Board and the Department of Veterans Affairs.

\subsection{Statistical Analysis}

We calculated descriptive statistics for study attributes and compared these attributes across the two publication eras using chi-squared or Wilcoxon rank-sum tests. Attributes with $\mathrm{p} \leq 0.1$ were included in a logistic regression model to identify study attributes that were associated with an increased likelihood of a negative outcome. For model interpretability, sample size was categorized as $200-900,1000-4999$, and $\geq 5000$. A final regression 
model was created using a stepwise-selection regression technique to identify attributes of RCT's that had an independent effect on the likelihood of a reported negative outcome. All analyses were performed using SAS Version 9.2 (SAS Institute Inc., Cary, NC, USA).

\section{Results}

We identified 1091 entries in the Cochrane Central Register published in the New England Journal of Medicine, The Lancet and the Journal of the American Medical Association in the pre-registration and registration eras. Of the 1091 entries, 181 (16\%) were excluded, leaving 917 reports of RCT's for our analysis (468 and 449 reports from the pre-registration and registration eras respectively, see Figure 1).

Descriptive statistics for reports of RCT's from each era are illustrated in Table 1. We found a high level of compliance with reporting of trial registration; $94.4 \%$ of trials in the registration era reported registration compared to no trials in the pre-registration era $(\mathrm{p}<0.001)$. There were more reports of drug trials in the registration era compared to the pre-registration era $(75.4 \%$ vs. $69.5 \%, p=0.02$ ), and there was a trend toward fewer reports of trials with procedural or device therapies in the registration era $(13.4 \%$ vs. $17.7 \%, \mathrm{p}=0.07)$. Compared to the pre-registration era, more RCT reports in the Registration era explicitly identified a primary endpoint $(56.0 \%$ vs. $69.5 \%, \mathrm{p} \leq 0.001)$ and more RCT's had non-inferiority primary endpoints $(4.7 \%$ vs $9.1 \%$, p $=$ 0.01). Median sample sizes were larger for RCT's reported in the registration era $(\mathrm{n}=750$ vs. $385, \mathrm{p} \leq$

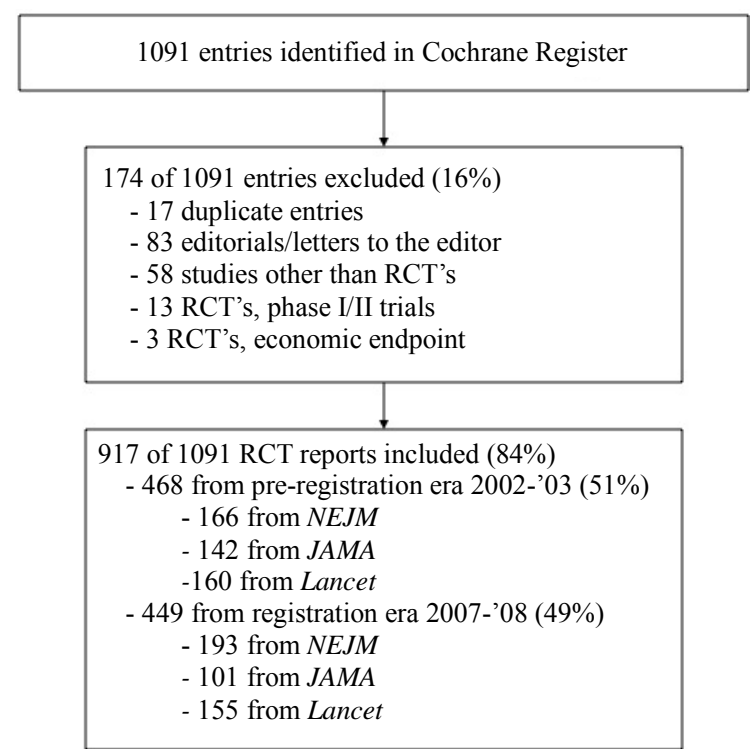

Figure 1. Flow Diagram of RCT reports reviewed for inclusion. Abbreviations: RCT, randomized controlled trial; NEJM, New England Journal of Medicine; JAMA, Journal of the American Medical Association.
$0.001)$. With regard to the primary outcome, $29.1 \%$ of RCT reports from the pre-registration era reported negative outcomes compared to $34.1 \%$ in the registration era. This difference did not meet statistical significance (Table $2, \mathrm{p}=0.10$ for unadjusted analysis, OR $1.26 \%, 95 \%$ CI 0.96 - 1.67). When analyzed by individual journal, a statistically significant difference was noted only for the New England Journal of Medicine, with a rate of negative publications of $24.1 \%$ in the pre-registration era and $35.2 \%$ in the registration era $(\mathrm{p}=0.02)$.

While publication era did not predict a differential likelihood of negative study outcomes, our logistic regression model did identify a number of factors associated with an increased or decreased likelihood of negative outcomes (Table 3). The five factors associated with an increased likelihood of a negative outcome were trials of drug therapies, trials of procedure or device therapies, explicit identification of a single primary endpoint, increasing sample size and discordance (discordant interpretation of the primary outcome by the two reviewers). The only factor associated with a decreased likelihood of

Table 1. Characteristics of RCT reports.

\begin{tabular}{|c|c|c|c|}
\hline Characteristic & $\begin{array}{c}\text { 2002-’03 } \\
\% \text { (n) }\end{array}$ & $\begin{array}{c}\text { 2007-’08 } \\
\text { \% (n) }\end{array}$ & p-value \\
\hline Registration reported & 0 & $94.4 \%(424)$ & $<0.0001$ \\
\hline Drug trial & $69.5 \%(325)$ & $75.4 \%(343)$ & 0.02 \\
\hline Procedure/device trial & $17.7 \%(83)$ & $13.4 \%(60)$ & 0.07 \\
\hline Surgery trial & $4.3 \%(20)$ & $2.7 \%(12)$ & 0.19 \\
\hline Behavioral trial & $10.9 \%(51)$ & $8.0 \%(36)$ & 0.14 \\
\hline Trial of diagnostic test & $2.4 \%(11)$ & $2.5 \%(11)$ & 0.92 \\
\hline Trial of treatment strategy & $13.0 \%(61)$ & $12.7 \%(57)$ & 0.88 \\
\hline Placebo controlled & $40.8 \%(191)$ & $42.3 \%(190)$ & 0.64 \\
\hline Non-inferiority design & $4.7 \%(22)$ & $9.1 \%(41)$ & 0.01 \\
\hline Single primary endpoint & $56.0 \%(262)$ & $69.5 \%(312)$ & $<0.0001$ \\
\hline Discordant & $10.3 \%(48)$ & $6.5 \%(29)$ & 0.04 \\
\hline U.S. correspondence address & $42.3 \%(198)$ & $39.0 \%(175)$ & $<0.0001$ \\
\hline Median sample size & 385 & 750 & \\
\hline
\end{tabular}

Table 2. Published RCT reports with negative outcomes, by era.

\begin{tabular}{cccc}
\hline Journal & 2002-’03 \% (n) & 2007-’08 \% (n) & p-value \\
\hline All journals & $29.1 \%(136)$ & $34.1 \%(153)$ & 0.10 \\
NEJM & $24.1 \%(40)$ & $35.2 \%(68)$ & 0.02 \\
JAMA & $38.7 \%(55)$ & $43.6 \%(44)$ & 0.45 \\
Lancet & $25.6 \%(41)$ & $26.5 \%(41)$ & 0.87 \\
\hline
\end{tabular}


Table 3. Logistic regression, modeling probability of a negative outcome (adjusted).

\begin{tabular}{cccc}
\hline & OR & 95\% CI & p-value \\
\hline $\begin{array}{c}\text { Publication era } \\
\text { (relative to pre-registration era) }\end{array}$ & 1.26 & $(0.93,1.71)$ & 0.14 \\
Drug trial & 1.62 & $(1.08,2.43)$ & 0.02 \\
Procedure/device trial & 2.08 & $(1.29,3.35)$ & 0.003 \\
Non-inferiority trial & .13 & $(0.05,0.31)$ & $<0.0001$ \\
Single primary endpoint & 1.79 & $(1.30,2.47)$ & 0.0004 \\
Discordant & 3.70 & $(2.13,6.25)$ & $<0.0001$ \\
Sample size & & & $<0.0001$ \\
200 - 999 relative to $<200$ & 1.80 & $(1.18,2.74)$ & \\
1000 - 4999 relative to $<200$ & 2.54 & $(1.61,4.00)$ & \\
$\geq 5000$ relative to $<200$ & 3.08 & $(1.78,5.34)$ & \\
Journal & & & $<0.0001$ \\
Lancet relative to NEJM & .82 & $(.58,1.18)$ & \\
JAMA relative to NEJM & 1.87 & $(1.29,2.71)$ & \\
\hline
\end{tabular}

Abbreviations: OR, odds ratio; CI confidence interval; NEJM, New England Journal of Medicine; JAMA, Journal of the American Medical Association

a negative outcome was a non-inferiority study design $(\mathrm{OR}=0.13, \mathrm{p}<0.001,95 \%$ CI $0.05-0.31)$.

\section{Discussion}

We proposed that the anecdotal perception of an increase in negative studies present in our academic journal club is a real effect and that it would be associated with mandatory clinical trial registration.

Our analysis did not find a significant difference in the proportion of published trials with negative outcomes between the two eras, even though there was a dramatic increase in reporting of trial registration, suggesting that trial registration may not have influenced the chances of negative trials being published in major medical journals. We also saw changes in the characteristics of reported trials. Sample sizes of published studies increased, climbing from a median of 385 to 750 . The percentage of reports explicitly identifying a primary outcome also increased. Improvement in clinical trial reporting was reflected practically in our finding that it became easier for abstracters to interpret trial outcomes, with discordant interpretation of trial outcomes (positive vs. negative) decreasing from the pre-registration era to the registration era. Though non-inferiority trials represented a small minority of published trials, the percentage of trials using a non-inferiority design nearly doubled across the two study eras. Use of a non-inferiority design was the only trial characteristic that was independently associated with a decreased likelihood of a negative outcome. Characteristics of trials that were associated with an increased likelihood of a negative reported outcome were drug or procedure interventions, larger sizes, and reporting of a single primary endpoint.

To our knowledge this study is the first to examine the effects of the ICMJE's mandatory clinical trial registration policy on publication of negative studies. Previous studies of publication bias have examined cohorts of trials from within specific institutions or registries, and have substantiated that negative trials are less likely to be published [2,3]. We chose to examine the question of publication bias at the level of the publishing journals, since the ICMJE's policy was enacted on this level and this is the level of exposure for the journal reader. We looked for evidence of changes in publication bias in the general medicine journals with the highest impact factors because research published in these journals is likely to be influential and because all of these journals are members of the ICMJE. In its 2004 policy statement, the ICMJE cites two primary drivers of publication biasinconclusive or negative results that are perceived as clinically uninformative and negative results that reflect poorly on a proprietary intervention [6]. The ICMJE's policy highlights efforts from participating journals to give appropriate weight to the importance of clinical trial results, regardless of whether these results are positive or negative. However, this policy will do little to affect publication bias within high-profile journals that is driven by clinically uninformative negative results; these trials are likely to be published in journals with lower impact factors. Our study was most likely to detect publication bias driven by negative results that reflected poorly on proprietary interventions. Other recent studies have demonstrated the connection between publication bias and selective outcomes reporting, where primary outcomes for trials of proprietary interventions appear to have been changed post hoc but prior to publication. $[2,10,11]$. Such pre-publication changes in reported outcomes may partly account for our own negative findings.

We did observe two study characteristics that may have driven this perceived increase in publication of negative trials. Surprisingly, the larger the trial the more likely it was to be negative.

This fact may represent studies in conditions that great treatment improvements have already been achieved and further therapies have marginal effect or therapies that have pushed the envelope too far past benefit to detriment. Also, the fact that more discordant studies were noted to be negative may be related to selective reporting. Negative trials may not have been as clear in their endpoints making study abstracters differ on their interpretation that the study was positive or negative. Only upon reviewing the full article was one able to determine 
whether the study's primary endpoint was truly negative.

Our study has a number of limitations. Our data are based primarily on reviews of article abstracts, which may not have contained the same information as full publications. Our sample size was not adequate to detect small differences between rates of publication of negative trials across the two study eras. Because we studied RCT reports from two eras, it was not possible to control fully for changes over time other than mandatory clinical trial registration. The effect of mandatory clinical trial registration on publication bias may be obscured by other trends in the medical literature, such as selective outcomes reporting or changes in the designs of randomized controlled trials that favor positive outcomes. We note that the editorial leadership at the three journals remained constant over the two study eras. Also, because we studied publication bias in the three general medicine journals with the highest impact factors, our results do not address changes in publication bias across the entire medical literature or within medical journals of lower impact factors.

Our study findings suggest that any effect of clinical trial registration on publication bias within top-tier medicine journals is likely to be small, if it exists. Other studies have shown substantial problems with selective outcomes reporting even among registered trials. Do these findings cast doubt on the utility of clinical trial registration as a tool to increase the transparency of clinical research? It seems probable that standards of adequacy for trial registration have not been sufficient $[12,13]$, and stricter enforcement of registration standards as well as new laws requiring minimal outcomes reporting may improve the utility of clinical trial registration to reduce publication bias and selective outcomes reporting.

Our findings also suggest that studies with non-inferiority designs are playing an increasingly important role in the medical literature. Non-inferiority trials nearly doubled as a proportion of all trials published in our study sample, and these trials are much more likely to have a positive outcome than trials with conventional superiority designs. While the ascendance of non-inferiority trials is a welcome trend in the arena of comparative effectiveness research, this trend may be less benign when applied in trials of proprietary therapies. In this latter arena, a non-inferiority trial design may provide a lower bar to evaluate the effectiveness of a therapy that is seeking a wider market share [14].

Future studies examining the rates of published negative trials and the effect of trial registration on publication bias should look for this effect in a broader range of journals, including subspecialty journals and smaller general medicine journals. Because publication bias may be driven by proprietary concerns, further inquiries should shed more light on the interaction between trial funding, publication bias and adoption of non-inferiority study designs.

In conclusion, our results do not substantiate an association between clinical trial registration and a reduction in publication bias in major medicine journals. Clinical trial registration is meant as a means toward maximizing the impact of clinical research activities and protecting the contributions of altruistic patients. As we become accustomed to the era of clinical trial registration (and now, mandatory outcomes reporting) it is imperative to learn what we can and cannot expect from this important tool.

\section{REFERENCES}

[1] R. J. Simes, "Publication Bias: The Case for an International Registry of Clinical Trials," Journal of Clinical Oncology, Vol. 4, No. 10, 1986, pp. 1529-1541.

[2] K. Dwan., D. G. Altman, J. A. Arnaiz, et al., "Systematic Review of the Empirical Evidence of Study Publication Bias and Outcome Reporting Bias," PLoS One, Vol. 3, No. 8, 2008, e3081. doi:10.1371/journal.pone.0003081

[3] S. Hopewell, K. Loudon, M. J. Clarke, A. D. Oxman and K. Dickersin, "Publication Bias in Clinical Trials Due to Statistical Significance or Direction of Trial Results," Cochrane Database of Systematic Reviews, Vol. 1, 2009, MR000006.

[4] K. Rising, P. Bachetti and L. Bero, "Reporting Bias in Drug Trials Submitted to the Food and Drug Administration: Review of Publication and Presentation," PLOS Medicine, Vol. 5, No. 11, 2008, e217.

doi:10.1371/journal.pmed.0050217

[5] K. Lee, P. Bachetti and I. Sim, "Publication of Clinical Trials Supporting Successful New Drug Applications: A Literature Analysis," PLoS Medicine, Vol. 5, No. 9, 2008, e191. doi:10.1371/journal.pmed.0050191

[6] C. D. Deangelis, J. M. Drazen, F. A. Frizelle, C. Haug, J. Hoey, R. Horton, S. Kotzin, C. Laine, A. Marusic, A. J. Overbeke, T. V. Schroeder, H. C. Sox and M. B. Van Der Weyden, "Clinical Trial Registration: A Statement from the International Committee of Medical Journal Editors," Journal of the American Medical Association, Vol. 292, No. 11, 2004, pp. 1363-1364. doi:10.1001/jama.292.11.1363

[7] D. A. Zarin, T. Tse and N. C. Ide, "Trial Registration at ClinicalTrials.gov between May and October 2005," New England Journal of Medicine, Vol. 353, No. 26, 2005, pp. 2779- 2787. doi:10.1056/NEJMsa053234

[8] Clinicaltrials.gov, "A Service of the US National Institutes of Health," 2010. http://www.clinicaltrials.gov

[9] ISI Web of Knowledge, “Journal Citation Reports,” 2010. http://0-admin-apps.isiknowledge.com.impulse.ucdenver. edu/JCR

[10] S. S. Vedula, L. Bero, R. W. Scherer and K. Dickersin, "Outcome Reporting in Industry-Sponsored Trials of Gabapentin for Off-Label Use," New England Journal of Medicine, Vol. 361, No. 20, 2009, pp. 1963-1971. 
doi:10.1056/NEJMsa0906126

[11] S. Mathieu, I. Boutron, D. Moher, D. Altman and P. Ravaud, "Comparison of Registered and Published Primary Outcomes in Randomized Controlled Trials," Journal of the American Medical Association, Vol. 302, No. 9, 2009, pp. 977-984. doi:10.1001/jama.2009.1242

[12] C. D. Deangelis, J. M. Drazen, F. A. Frizelle, C. Haug, J. Hoey, R. Horton, S. Kotzin, C. Laine, A. Marusic, A. J. Overbeke, T. V. Schroeder, H. C. Sox and M. B. Van Der Weyden, "Is This Clinical Trial Fully Registered? A Statement from the International Committee of Medical Journal Editors," New England Journal of Medicine, Vol.
352, No. 23, 2005, pp. 2436-2438. doi:10.1056/NEJMe058127

[13] J. S. Ross, G. K. Mulvey, E. M. Hines, S. E. Nissen and H. M. Krumholz, "Trial Publication after Registration in ClinicalTrials.gov: A Cross-Sectional Analysis," PLoS Medicine, Vol. 6, No. 9, 2009, e1000144. doi:10.1371/journal.pmed.1000144

[14] S. Garatini and V. Bertele, "Non-Inferiority Trials Are Unethical Because They Disregard Patients' Interests," Lancet, Vol. 370, No. 9602, 2007, pp. 1875-1877. doi:10.1016/S0140-6736(07)61604-3 